\title{
Effect of RNA interference of the expression of HMGA2 on the proliferation and invasion ability of ACHN renal cell carcinoma cells
}

\author{
YING LIU ${ }^{1,2}$, QI-ZHONG FU ${ }^{2}$, LIN PU ${ }^{2}$, LING-LING SONG ${ }^{2}$, YI-YUN WANG ${ }^{2}$, \\ JING LIU ${ }^{2}$, ZHEN-LONG WANG ${ }^{3}$ and ZI-MING WANG ${ }^{3}$
}

\begin{abstract}
${ }^{1}$ Xi'an Jiaotong University Health Science Center, Xi'an, Shanxi 710049; ${ }^{2}$ Department of Urological Surgery, The Affiliated Zhongshan Hospital of Dalian University, Dalian, Liaoning 116001; ${ }^{3}$ Department of Urological Surgery, The Second Affiliated Hospital of Xi'an Jiaotong University, Xi'an, Shanxi 710004, P.R. China
\end{abstract}

Received May 10, 2016; Accepted April 4, 2017

DOI: $10.3892 / \mathrm{mmr} .2017 .7258$

\begin{abstract}
This aim of the present study was to observe the effect of high mobility group AT-hook 2 (HMGA2) on the proliferation and invasion ability of ACHN renal cell carcinoma (RCC) cells. Human ACHN cells, an RCC cell line, and HKC normal human renal tubular epithelial cells were cultured. HMGA2 small interfering (si)RNA, Mock-siRNA and their negative control group were designed and synthesized. Subsequently, the ACHN cells were transiently transfected using RNA interference technology. Finally, the mRNA and protein expression levels of HMGA2 were detected using reverse transcription-polymerase chain reaction and western blot analyses. The proliferation ability of the ACHN cells was determined using MTT, and ACHN cell invasion ability was detected using the Transwell method. The results showed that the mRNA and protein expression levels of HMGA2 in the ACHN cells were considerably higher, compared with those in the HKC cells $(\mathrm{P}<0.01)$. The RCC cells, in which the expression of HMGA2 was specifically silenced, was successfully constructed. The proliferation rate of cells in the HMGA2-siRNA group was significantly lower, compared with that of cells in the Mock-siRNA group and control group at $24,48,72$ and $96 \mathrm{~h}$ post-transfection $(\mathrm{P}<0.05)$. The invasion ability of cells in the HMGA2-siRNA group was significantly lower, compared with that of cells in the Mock-siRNA group and control group $(\mathrm{P}<0.05) 48 \mathrm{~h}$ following transfection. Therefore, the HMGA2 gene may function as an oncogene in the occurrence and development of RCC, and provide specific targets for the targeted therapy of RCC. Further detailed
\end{abstract}

Correspondence to: Dr Zi-Ming Wang, Department of Urological Surgery, The Second Affiliated Hospital of Xi'an Jiaotong University, 157 West Fifth Road, Xi'an, Shanxi 710004, P.R. China E-mail: zimingwangdoc@163.com

Key words: renal cell carcinoma, high mobility group AT-hook 2, RNA interference, proliferation, invasion investigations of the HMGA2 gene are important for future gene therapy of RCC.

\section{Introduction}

High mobility group AT-hook 2 (HMGA2) is a non-histone protein combined with the chromosome. It lacks transcriptional activity, however, it binds with DNA leading to alterations in the structure of chromatin, and causing it to bend, stretch, curl, cyclize or unwind, This regulates the transcription of target genes, therefore, it is also known as an architectonic transcription factor (1). HMGA2 is important in the occurrence, invasion and metastasis of tumors; and is one of the cancer genes to received attention over previous years (2). Our previous study revealed that HMGA2 was expressed at a high level in RCC tissues, compared with renal benign tumors and normal kidney tissues, in which its expression was low or absent. Therefore, HMGA2 has become a useful novel pathological marker of RCC (3). In order to further investigate the effect of HMGA2 in RCC cells, the present study transfected ACHN cells with specific small interfering (si)RNA using RNA interference technology, and investigated alterations in the proliferation and invasion abilities of ACHN cells when the gene expression of HMGA2 was specifically silenced.

\section{Materials and methods}

Materials. ACHN cells, an RCC cell line, and the normal HKC cell line were purchased from the Cell Bank of the Institute of Basic Medical Sciences, Chinese Academy of Medical Sciences, Peking Union Medical College (Beijing, China). The TRIzol kit was purchased from Invitrogen; Thermo Fisher Scientific, Inc. (Waltham, MA, USA). Mouse anti-human HMGA2 monoclonal antibody (cat. no: GTX100519) was purchased form GeneTex, Inc. (Irvine, CA, USA) and the secondary goat anti-mouse IgG antibody (cat no: MBS176679) was from MyBioSource, Inc. (San Diego, CA, USA). Reverse transcription kits with 2X PCR Master Mix Taq were purchased from Beijing Tiangen Biotech Co., Ltd. (Beijing, China). Primers were purchased from Beijing 
Aoke Biotechnology Co., Ltd. (Beijing, China). The bicinchoninic acid (BCA) protein assay kit was purchased from Shenyang Wanlei Biological Technology Co. Ltd. (Shenyang, China) HMGA2-siRNA and Mock-siRNA were purchased from Shanghai GenePharma (Shanghai, China). MTT was purchased from Shanghai Sangon Biotech (Shanghai, China). Transwell filters were purchased from BD Biosciences (Franklin Lakes, NJ, USA).

Cell culture. The ACHN RCC cells and HKC cells were grown in RPMI-1640 culture medium with $10 \%$ fetal bovine serum (FBS; Gibco; Thermo Fisher Scientific, Inc.), and cultured at $37^{\circ} \mathrm{C}$ under $5 \% \mathrm{CO}_{2}$ conditions until the cells grew and adhered to the wall at days 2-3 for one generation.

Transient transfection. The transient transfection of cells was performed according to the manufacturer's protocol of the Lipofectamine 2000 reagent transfection kit. Two autoclaved EP tubes were used, one of which contained $10 \mu \mathrm{l}$ of siRNA $+240 \mu \mathrm{l}$ of serum-free RPMI-1640, and the other contained $10 \mu \mathrm{l}$ of Lipofectamine $+240 \mu \mathrm{l}$ of serum-free RPMI-1640. The contents of the two tubes were gently mixed, following which the mixture was incubated at $22^{\circ} \mathrm{C}$ temperature for $20 \mathrm{~min}$ to form a siRNA-Lipofectamine complex. The complex liquid containing liposome and siRNA was gradually added into the cell bottle (cell confluence, 80-90\%), and was incubated in an incubation chamber. After $24 \mathrm{~h}$, RNA was extracted for reverse transcription-polymerase chain reaction (RT-PCR) analysis and, after $48 \mathrm{~h}$, protein was extracted for western blot analysis.

Detection of the mRNA expression of HMGA2 using RT-PCR analysis. Total RNA was extracted using a TRIzol one-step method and the concentration of the RNA sample was determined using a spectrophotometer. Subsequently, $5 \mu \mathrm{l}$ of the RNA sample was obtained and placed on a $1.0 \%$ agarose gel (with $0.5 \mu \mathrm{g} / \mathrm{ml}$ of ethidium bromide) for gel electrophoresis, to identify the integrity of the RNA sample. Subsequently, $3 \mu \mathrm{g}$ of RNA was reverse transcribed into cDNA. These specific steps were performed in accordance with the kit instructions (cat. no. CW0581; Invitrogen; Thermo Fisher Scientific, Inc.). HMGA2-specific primer sequences were designed according to published literature (4), and semi-quantitative PCR analysis was performed (reaction contained: $5 \mathrm{X}$ PrimeScriPt ${ }^{\mathrm{TM}}$ Buffer, $4 \mu \mathrm{l}$; PrimeScriPt ${ }^{\mathrm{TM}}$ RT Enzyme mix I, $1 \mu \mathrm{l} ; 50 \mu \mathrm{M}$ Oligo dT primer, $1 \mu \mathrm{l} ; 100 \mu \mathrm{M}$ random 6mers, $1 \mu \mathrm{l}$; total RNA, $1 \mu \mathrm{g}$ ). The primer sequences were as follows: Forward 5'-CGAAAGGTGCTGGGCAGCTCCGG-3' and reverse 5'-CCATTTCCTAGGTCTGCCTCTTG-3', with an amplified fragment length of $323 \mathrm{bp}$; and $\beta$-actin, forward 5'-GAAGGTGAAGGTCGGAGTC-3' and reverse 5'-GAA GATGGTGATGGGATTTC-3', with an amplified fragment length of $228 \mathrm{bp}$. The reaction conditions of cDNA amplification using PCR were as follows: Pre-degeneration at $95^{\circ} \mathrm{C}$ for $3 \mathrm{~min}$, degeneration at $93^{\circ} \mathrm{C}$ for $30 \mathrm{sec}$, annealing at $58^{\circ} \mathrm{C}$ for $30 \mathrm{sec}$, and extension at $72^{\circ} \mathrm{C}$ for $30 \mathrm{sec}$, in a total of 30 cycles. This was subsequently followed by $10 \mathrm{~min}$ of extension, and finally stored at $4^{\circ} \mathrm{C}$. The reaction volume was $25 \mu \mathrm{l}$. The PCR amplification products underwent gel electrophoresis on $1.5 \%$ agarose (with $0.5 \mu \mathrm{g} / \mathrm{ml}$ of ethidium bromide), and the results were analyzed using the BioSpectrum Imaging System (UVP, Inc., Upland, CA, USA) for gel imaging analysis.

Detection of the protein expression of HMGA2 using western blot analysis. At $48 \mathrm{~h}$ following transfection of the ACHN cells with HMGA2-siRNA or Mock-siRNA, respectively, the cells were collected and lysed. Total cellular protein was extracted and underwent protein quantification using the bicinchoninic acid method. Proteins $(5 \mu \mathrm{g})$ were separated by 17\% SDS-PAGE under a constant current of $200 \mathrm{~mA}$ for $2 \mathrm{~h}$, and the protein was transferred onto a polyvinylidene fluoride film. The film was incubated in TBST containing 5\% skimmed milk powder for $1 \mathrm{~h}$, following which the mouse anti-human HMGA2 monoclonal primary antibody was added (1:1,000 dilution) and incubated overnight at $4^{\circ} \mathrm{C}$. The film was washed in TBST three times, following which the secondary goat anti-mouse $\operatorname{IgG}$ antibody was added (1:3,000 dilution) and incubated at room temperature for $1 \mathrm{~h}$, The film was washed in TBST three times and the results were analyzed using the Chem Image 5500 gel imaging system. The internal control antibody was $\beta$-actin (1:1,000; A01010; Abbkine Scientific, Co., Ltd., Wuhan, China ) mouse anti-human monoclonal antibody.

Detection of the proliferation of RCC cells using MTT. Cells in the logarithmic growth phase were collected, trypsinized and counted. A 96-well plate was used with $200 \mu 1$ wells. The peripheral wells were filled with PBS, and the cells $\left(1 \times 10^{4}\right.$ cells/well) were cultured at $37^{\circ} \mathrm{C}$ under $5 \% \mathrm{CO}_{2}$ conditions. When the cells had attached to the wall, transient transfection was performed according to the above-mentioned method. At 24, 48, 72 and 96 h post-transfection, freshly prepared MTT solution $(5 \mathrm{mg} / \mathrm{ml})$ was added, respectively, at a volume of $20 \mu \mathrm{l} /$ well. Following incubation at $37^{\circ} \mathrm{C}$ for $4 \mathrm{~h}$, the supernatant in the wells was carefully removed and discarded. DMSO $(0.5 \mathrm{mg} / \mathrm{ml})$ was added $(150 \mu \mathrm{l} /$ hole $)$, and the mixture was agitated for $10 \mathrm{~min}$ on a horizontal rotator in the dark for the crystals to fully dissolve. The absorbance value of each well was determined using an enzyme microplate reader at a $490 \mathrm{~nm}$ wavelength. The calibration wavelength was $>650 \mathrm{~nm}$, the blank control well was adjusted to zero, and the mean value of three repeats was determined. With time as the horizontal axis and absorbance value as the vertical axis, the cell growth curve was drawn.

Detection of the invasion ability of RCC cells using a Transwell assay. The cells were collected in the logarithmic growth phase, washed twice with PBS, trypsinized, centrifuged at $400 \times \mathrm{g}$ at $37^{\circ} \mathrm{C}$ for $5 \mathrm{~min}$. and re-suspended in serum-free PRMI-1640. The mixture underwent dissociated preparation for single cell suspension, and the number of cells was adjusted to $\sim 5 \times 10^{5} / \mathrm{ml}$. Following the addition of Matrigel to serum-free RPMI-1640, $100 \mu \mathrm{l}$ of the single cell suspension was obtained and transferred to the upper compartment of the Transwell chamber; whereas the lower compartment was filled with $500 \mu \mathrm{l}$ of RPMI-1640 containing 10\% FBS. Following $48 \mathrm{~h}$ of conventional culture, the Transwell chamber was removed and washed twice with PBS, and the Matrigel above the chamber, containing cells attached to the polycarbonate film, were wiped off with a cotton swab. The Transwell chamber was placed in 


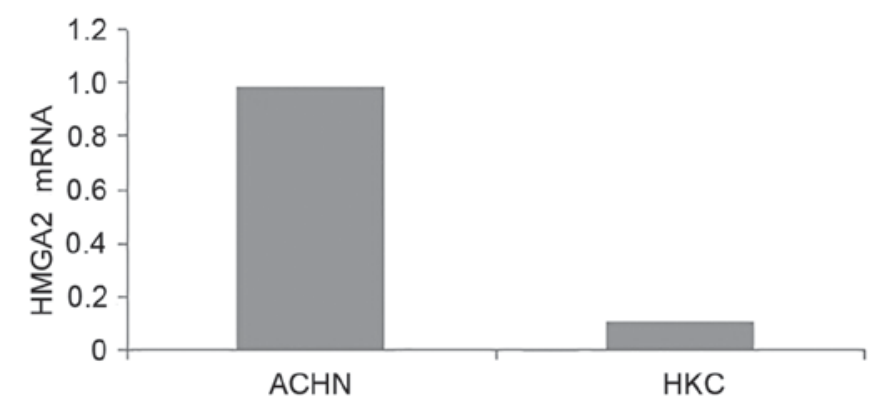

Figure 1. Relative mRNA expression of HMGA2. $\mathrm{P}<0.01$ between the ACHN and HKC cells. HMGA2, high mobility group AT-hook 2.
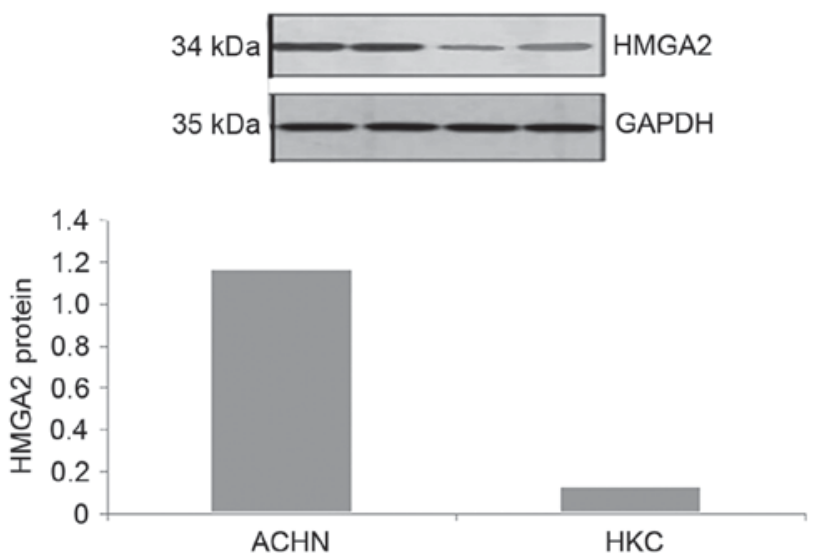

Figure 2. Relative protein expression of HMGA2. $\mathrm{P}<0.01$ between the ACHN and HKC cells. HMGA2, high mobility group AT-hook 2.

4\% polyformaldehyde, fixed for $20 \mathrm{~min}$, removed and dried, and placed in a crystal violet solution at room temperature for 10 min. The excessive crystal violet was removed with PBS followed by air-drying. Under the view of a high power light microscope, five fields were randomly selected and the number of cells was counted.

Statistical analysis. Statistical analysis was performed using SPSS 17.0 statistical software (SPSS, Inc., Chicago, IL, USA). Comparisons of the differences between the experimental group and control group were performed using a Student's t-test, and multiple comparisons between groups were performed using one-way analysis of variance, followed by Fisher's least significant difference test. $\mathrm{P}<0.05$ was considered to indicate a statistically significant difference.

\section{Results}

Expression of HMGA2 in human RCC cells and HKC cells. Using RT-PCR analysis, the mRNA expression levels of HMGA2 in the human RCC ACHN cells and human HKC cells were $0.98 \pm 0.16$ and $0.10 \pm 0.03$, respectively. The expression in the ACHN cells was higher, compared with that in the HKC cells $(t=8.92 ; \mathrm{P}<0.01)$ with statistical significance (Fig. 1 ).

Using western blot analysis, the protein levels of HMGA2 in human RCC ACHN cells and HKC cells were 1.16 \pm 0.20 and $0.12 \pm 0.05$, respectively. The expression level in the ACHN

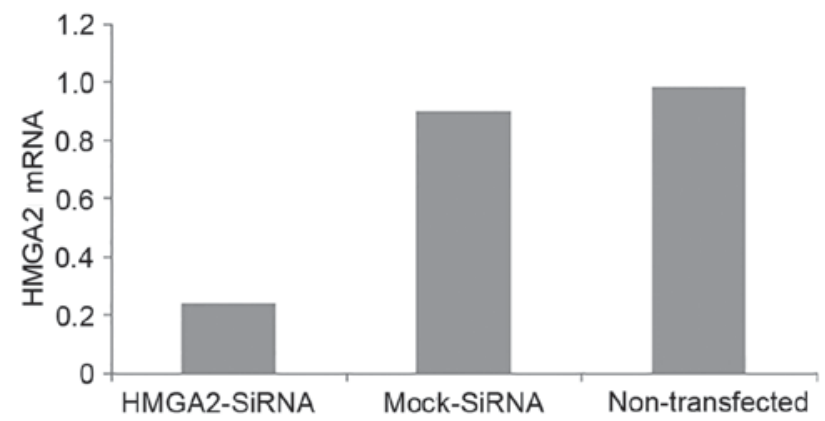

Figure 3. Relative mRNA expression of HMGA2 following siRNA interference. $\mathrm{P}<0.01$ between the HMGA2-siRNA and non-transfected cells. HMGA2, high mobility group AT-hook 2; siRNA, small interfering RNA.
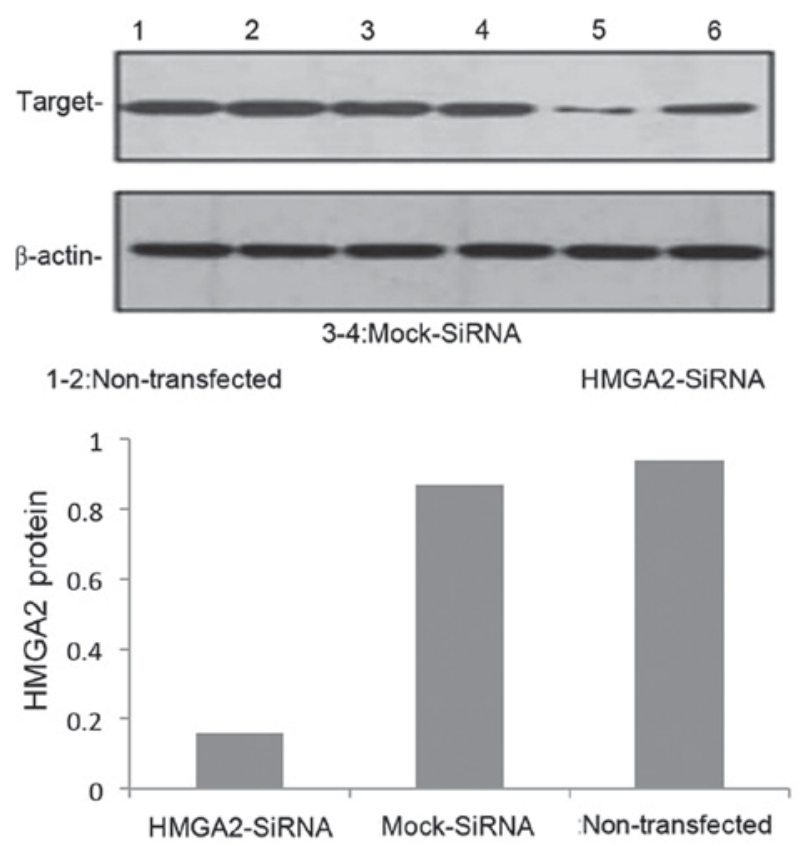

Figure 4. Protein expression of HMGA2 following siRNA interference. $\mathrm{P}<0.01$ between the HMGA2-siRNA and non-transfected cells. HMGA2, high mobility group AT-hook 2; siRNA, small interfering RNA.

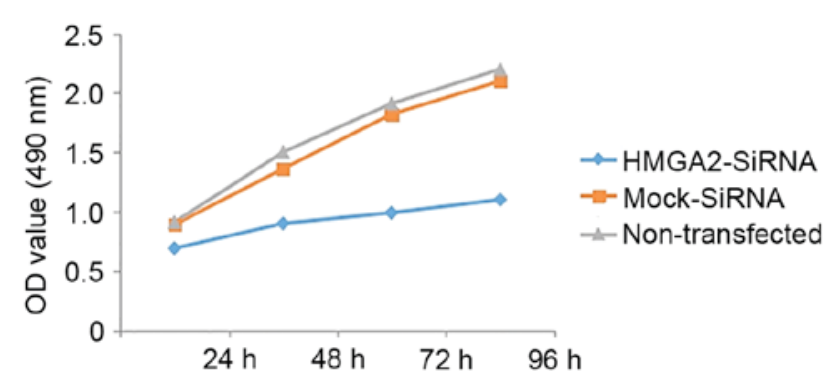

Figure 5. Alterations in the proliferation ability of ACHN cells following siRNA interference. $\mathrm{P}<0.05$ between the HMGA2-siRNA and non-transfected cells. HMGA2, high mobility group AT-hook 2; siRNA, small interfering RNA; OD, optical density.

cells was higher, compared with that in the HKC cells. $(\mathrm{t}=9.21$; $\mathrm{P}<0.01$ ) with statistical significance (Fig. 2).

Expression of HMGA2 in ACHN cells following siRNA interference. At $24 \mathrm{~h}$ following transfection with HMGA2-siRNA 
Table I. Comparison of cell proliferation ability at different time points.

\begin{tabular}{lcccc}
\hline Group & $24 \mathrm{~h}$ & $48 \mathrm{~h}$ & $72 \mathrm{~h}$ & $96 \mathrm{~h}$ \\
\hline HMGA2-siRNA & $0.69 \pm 0.02$ & $0.90 \pm 0.05$ & $0.99 \pm 0.07$ & $1.10 \pm 0.09$ \\
Mock-siRNA & $0.89 \pm 0.03$ & $1.36 \pm 0.06$ & $1.82 \pm 0.08$ & $2.10 \pm 0.10$ \\
Non-transfected & $0.91 \pm 0.02$ & $1.50 \pm 0.06$ & $1.91 \pm 0.10$ & $2.20 \pm 0.12$ \\
\hline
\end{tabular}

Values are presented as the mean \pm standard deviation. $\mathrm{P}<0.05$, compared with the Mock-siRNA transfection group and non-transfected group. HMGA2, high mobility group AT-hook 2; siRNA, small interfering RNA.

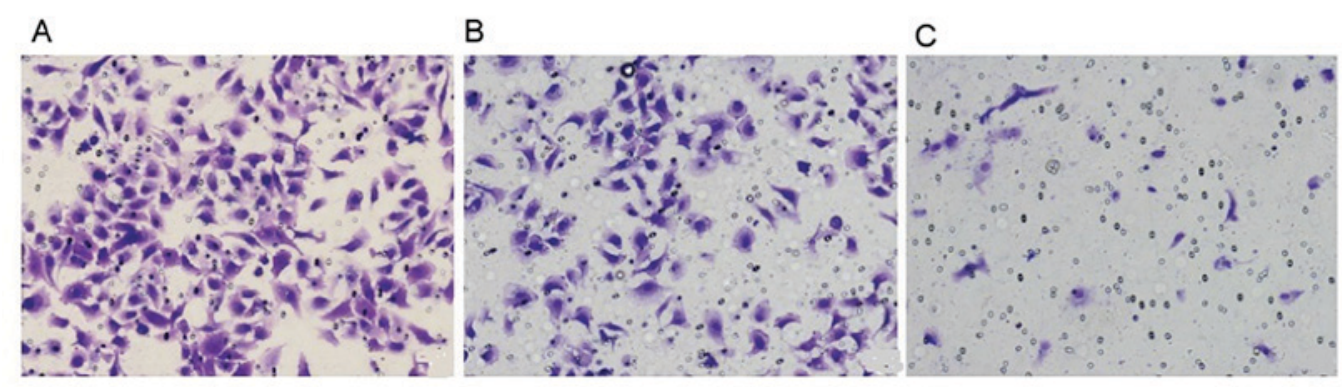

Figure 6. Alterations in the invasion ability of ACHN cells following siRNA interference. P<0.05 between the HMGA2-siRNA and non-transfected cells. (A) non-transfected group, (B) mock-siRNA transfection group, (C) HMGA2-siRNA transfection group. HMGA2, high mobility group AT-hook 2; siRNA, small interfering RNA. Magnification, x400.

and Mock-siRNA, respectively, the cells were collected, total RNA was extracted, reverse transcription was performed and the mRNA expression of HMGA2 was detected using RT-PCR analysis. The results showed that the mRNA levels in the HMGA2-siRNA, Mock-transfection and control groups were $0.24 \pm 0.08,0.90 \pm 0.12$ and $0.98 \pm 0.16$, respectively. The mRNA expression of HMGA2 in the HMGA2-siRNA transfection group was significantly lower, compared with the levels in the Mock-siRNA transfection group and control group $(\mathrm{t}=5.28$; $\mathrm{P}<0.01$; Fig. 3).

At $24 \mathrm{~h}$ post-transfection with HMGA2-siRNA and Mock-siRNA, respectively, the cells were collected, total protein was extracted, and alterations in the protein expression levels of HMGA2 were detected using western blot analysis. It was found that the protein expression of HMGA2 in the HMGA2-siRNA transfection group was significantly lower, compared with the levels in the Mock-siRNA transfection group and control group (Fig. 4). These results confirmed that, following siRNA interference, the mRNA and protein expression levels of HMGA2 were significantly reduced, suggesting successful interference and a high level of siRNA specificity.

Alterations in the proliferation ability of ACHN cells following siRNA interference. The proliferation ability of ACHN cells was detected using MTT 24, 48, 72 and $96 \mathrm{~h}$ following siRNA interference, respectively. Compared with cells in the Mock-siRNA transfection group and control group, ACHN cells in the HMGA2-siRNA transfection group had decreased proliferation ability, and this difference was statistically significant $(\mathrm{P}<0.05)$. No significant difference was found between the Mock-siRNA transfection group and control group (P>0.05; Table I and Fig. 5).

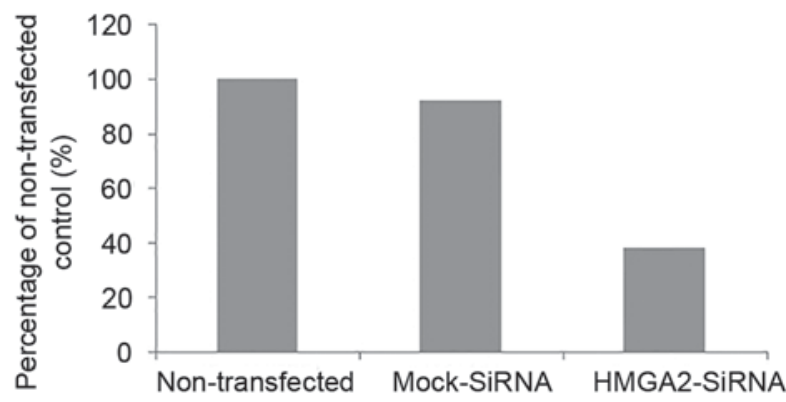

Figure 7. Alterations in the invasion ability of ACHN cells following siRNA interference. $\mathrm{P}<0.05$ between the HMGA2-siRNA transfection group and the non-transfected group. HMGA2, high mobility group AT-hook 2; siRNA, small interfering RNA.

Alterations in the invasion ability of ACHN cells following siRNA interference. The invasion ability of ACHN cells was detected using a Transwell chamber $48 \mathrm{~h}$ following siRNA interference. Compared with cells in the Mock-siRNA transfection group and control group, ACHN cells in the HMGA2-siRNA transfection group exhibited decreased invasion ability, and this difference was statistically significant $(\mathrm{P}<0.05$; Figs. 6 and 7$)$.

\section{Discussion}

RNA interference is a type of post-transcriptional gene silencing and is described as when, in double-stranded RNA with homologous complementary sequences of specific mRNA, the transcriptional product of the target gene is introduced into the cell and the specific mRNA is specifically 
degraded, resulting in a corresponding lack of function phenotype (5). This provides an effective gene-inhibiting technology with high specificity (5). At present, RNA interference has been widely used in investigations of gene function, signal transduction pathways and gene therapy (6). In previous years, siRNA technology has enabled progress in tumor gene therapy by introducing plasmid or viral vectors, which express siRNA, into tumor cells and allowing the examination of specific oncogenes or anti-oncogene functions (7).

The HMGA2 gene is located in the q15 area of human chromosome 12, which consists of five exons and four introns. Among these, the first, second and third exon encode three DNA-binding regions. The fourth exon encodes a spacer region between the DNA-binding region and the acidic carboxy terminal, and the fifth exon encodes the acidic carboxy terminal (8). As a structural transcription factor, HMGA2 can regulate the transcription and activation of a large number of genes, particularly those associated with cell proliferation and apoptosis (9). HMGA2 is important in the occurrence, invasion and metastasis of tumors (10). In previous years, significant correlations between HMGA2 and epithelial-mesenchymal transition, microRNA and stem cells have become a focus in basic and clinical investigations of tumors (11). Our previous study (3) revealed that the mRNA and protein expression levels of HMGA2 in RCC tissues are significantly higher, compared with those in benign renal tumor tissues and normal renal tissues; and the expression of HMGA2 is significantly correlated with tumor-node-metastasis (TNM) staging and lymph node metastasis of RCC. The higher the TNM stage of the tumor, the higher the expression of HMGA2, and the shorter the survival rates of patients (3). Therefore, HMGA2 may act as oncogene in the development of RCC. Tumor invasion and metastasis are the leading causes of mortality in patients with RCC; and surgical resection continues to be the primary treatment method for RCC (12). However, studies have revealed that $30 \%$ of patients with RCC develop recurrence or metastasis following surgery (13). The invasion and metastatic ability of RCC cells is a key factor in the reason RCC is difficult to cure completely (14). Therefore, based on previous studies, the present study further examined the effect of the HMGA2 gene on the biological characteristics of RCC cells (15).

In the present study the mRNA and protein expression levels of HMGA2 were detected in human RCC ACHN cells and HKC cells using RT-PCR and western blot analyses. It was found that the expression of HMGA2 in ACHN cells was significantly higher, compared with that in HKC cells, which provided a foundation for subsequent experiments. Using RNA interference technology, the ACHN cells were transfected with HMGA2-siRNA or Mock-siRNA. Following transfection, the mRNA and protein expression levels of HMGA2 in the ACHN cells were significantly decreased, indicating that the gene silencing had been successful and the specificity of HMGA2-siRNA was high. An MTT assay was performed 24, 48, 72 and 96 h post-transfection, respectively, to detect alterations in proliferation ability. The results showed that, at $24 \mathrm{~h}$ post-transfection, the proliferation ability of the ACHN cells transfected with HMGA2-siRNA was significantly decreased, compared with the Mock-siRNA transfection and non-transfected groups. These results indicated that HMGA2 enhanced the proliferation of RCC cells.
The primary contributor to rates of mortality in patients with malignant tumors is the proliferation of tumor cells and the second, which is also important, is the invasion of tumor cells (16). In the present study, alterations in the invasion ability of ACHN cells were examined following transfection with HMGA2-siRNA. At $48 \mathrm{~h}$ post-siRNA interference, the invasion ability of the ACHN cells was detected using a Transwell chamber assay. The results showed that the invasion ability of ACHN cells transfected with HMGA2-siRNA was significantly decreased, compared with the ACHN cells transfected with Mock-siRNA and the non-transfected cells, indicating that HMGA2 enhanced the invasion ability of the RCC cells.

The function of RNA molecules with 20-30 nucleotides in the genome have become an increasing focus of investigations. Among RNA molecules, siRNAs and microRNAs (miRNAs) are the two primary categories. They are not only involved in the regulation of important biological activities, including growth, development and apoptosis and immunity $(17,18)$; but are also closely associated with the occurrence and metastasis of malignant tumors (19). This has revealed that the non-encoding region of a gene may have important functions. In a comparative study, Cheng et al found that the expression of three types of miRNA (miR-34a, miR-21 and miR-224) were upregulated in RCC tissues, compared with normal kidney tissue (20). A similar gene-silencing effect was found in miRNA, as in siRNA. The results of the present study revealed that the proliferation and invasion ability of RCC cells was significantly enhanced when the expression of HMGA2 was silenced using siRNA. Therefore, there may be certain types of miRNA in patients with RCC, which can target the regulation of HMGA2, and promote cancer tumorigenesis and metastasis. However, the specific molecular mechanism of the positive regulation of HHMGA2 remains to be elucidated. Therefore, detailed investigations of the specific roles and signal transduction mechanism of HMGA2 in the development of RCC may provide a new theoretical basis and direction for gene therapy of RCC.

\section{References}

1. Reeves R and Beckerbauer L: HMGI/Y proteins: Flexible regulators of transcription and chromatin structure. Biochim Biophys Acta 1519: 13-29, 2011.

2. Hammond SM and Sharpless NE: HMGA2 microRNA, and stem cell aging. Cell 135: 1013-1016, 2008.

3. Liu Y, Fu QZ, Pu L, Meng QG, Liu XF, Dong SF, Yang JX and Lv GY: HMGA2 expression in renal carcinoma and its clinical significane. J Med Biochem 34: 338-343, 2015.

4. Wang X, Liu X, Li AY, Chen L, Lai L, Lin HH, Hu S, Yao L, Peng J, Loera $\mathrm{S}$, et al: Overexpression of HMGA2 promotes metastasis and impacts survival of colorectal cancers. Clin Cancer Res 17: 2570-2580, 2011.

5. Timmons L, Court D and Fire A: Ingestion of bacterially expressed dsRNAs can produce specific and potent genetic interference in Caenorhabditis elegans. Gene 263: 103-112, 2001.

6. Lu ZJ and Mathew DH: Efficient siRNA selection using hybridization thermodynamics. Nucleic Acids Res 36: 640-647, 2008.

7. Yu JY, DeRuiter SL and Turner DL: RNA interference by expression of short-interfering RNAs and hairpin RNAs in mammalian cells. Proc Natl Acid Sci USA 99: 6047-6052, 2002.

8. Medeiros F, Ataujo AR, Erickson-Johnson MR, Kashyap PC, Dal Cin P, Nucci M, Wang X, Bell DA and Oliveira AM: HMGA1 and HMGA2 rearrangements in mass-forming endometriosis. Genes Chromosomes Cancer 49: 630-634, 2010. 
9. Li AY, Boo LM, Wang SY, Lin HH, Wang CC, Yen Y, Chen BP, Chen DJ and Ann DK: Suppression of nonhomolognus and joining repair by overexpression of HMGA2. Cancer Res 69: 5699-5706, 2009.

10. Borrmann L, Schwanbeck R, Heyduk T, Seebeck B, Rogalla P, Bullerdiek J and Wisniewski JR: High mobility group A2 protein and its derivatives bind a specific region of the promoter of DNA repair gene ERCCI and medulate its activity. Nucleic Acids Res 31: 6841-6851, 2003.

11. Nishino J, Kim I, Chada K and Morrison SJ: Hmga2 promotes neural stem cell self-renewal in young but not old mice by reducing p16Ink4a and p19Arf Expression. Cell 135: 227-239, 2008.

12. Frank I, Blute ML, Cheville JC, Lohse CM, Weaver AL and Zincke H: An outcome prediction model for patients with clear cell renal cell carcinoma treated with radical nephrectomy based on tumor stage, size, grade and necrosis: The SSIGN score. J Urol 68: 2395-2400, 2002.

13. Van Spronsen DJ, Mulders PF and De Mulder PH: Novel treatments for metastatic renal cell carcinoma. Crit Rev Oncol Hematol 55: 177-191, 2005.

14. Thyavihally YB, Mahantshetty U, Chamarajanagar RS, Raibhattanavar SG and Tongaonkar HB: Management of renal cell carcinoma with solitary metastasis. World J Surg Oncol 3: 48, 2005.
15. Frank I, Blute ML, Cheville JC, Lohse CM, Weaver AL and Zincke H: An outcome prediction model for patients with clear cell renal cell carcinoma treated with radical nephrectomy based on tumor stage, size, grade and necrosis: The SSIGN score. J Urol 168: 2395-2400, 2002.

16. Inoué S: Microtubule dynamics in cell division: Exploring living cells with polarized light microscopy. Annu Rev Cell Dev Biol 24: 1-28, 2008.

17. Zeng Y and Cullen BR: Sequence requirements for micro RNA processing and function in human cells. RNA 9: 112-123, 2003.

18. Nolasco S, Bellido J, Gonçalves J, Zabala JC and Soares H: Tubulin factor A gene silencing in mammalian cells induces changes in microtubule cytoskeleton, cell cycle arrest and cell death. FEBS Lett 579: 3515-3524, 2005.

19. Nana-Sinkam SP and Croce CM: C1inical applications for microRNA in cancer. Clin Pharmacol Ther 93: 98-104, 2013.

20. Cheng T, Wang L, Li Y, Huang C, Zeng L and Yang J: Differential microRNA expression in renal cell carcinoma. Oncol Lett 6: 769-772, 2013. 